\title{
Coffee consumption and hip fracture risk: a meta-analysis
}

\author{
Xin-li $\mathrm{Li}^{1} * \dagger$ and Jiu-hong $\mathrm{Xu}^{2} \dagger$ \\ ${ }^{1}$ School of Public Health, Medical College of Soochow University, Suzhou 215123, Jiangsu, People's Republic of China \\ ${ }^{2}$ Department of Radiotherapy, The First Affiliated Hospital of Soochow University, Surhou 215006, Jiangsu, People's Republic of China
}

(Received 6 November 2012 - Final revision received 15 April 2013 - Accepted 19 April 2013)

Journal of Nutritional Science (2013), vol. 2, e23, page 1 of 8

doi:10.1017/jns.2013.13

Abstract

To investigate the effect of coffee consumption on hip fracture risk, a meta-analysis was conducted. The PubMed database was screened for all published studies about coffee consumption and hip fracture through to November 2011. Reviews, PubMed option 'related articles' and references of retrieved papers were also searched for potentially relevant papers. Only studies that contained OR with $95 \%$ CI for the association between coffee consumption and hip fracture risk were included. The summary risk estimates were calculated by fixed- and random-effects models. Subgroup analyses were carried out stratified by study designs and participant characteristics, respectively. A total of six prospective cohort studies and six case-control studies were included in the final analysis. The pooled OR displayed increased risk of hip fracture by $29.7 \%$ (95\% CI 0.960, 1.751; $P=0.09)$ for the highest compared with the lowest coffee consumption by the random-effects model ( $P$ for heterogeneity $=0.000 ; I^{2}=84.0 \%$ ), but the result had no statistical significance. Subgroup analyses showed that coffee consumption significantly increased hip fracture risk by $54 \cdot 7 \%$ (95\% CI $1 \cdot 152,2 \cdot 077 ; P=0 \cdot 004)$ among women, by $40 \cdot 1 \%$ (95\% CI 1.015, 1.935; $P=0.040)$ for elderly participants aged over 70 years, and by $68.3 \%$ for Northern Americans (95 \% CI $1.492,1.899 ; P=0.000)$. Other subgroup analyses according to data published before the year 2000 showed a positive association between coffee and hip fracture risk, and followup duration also positively affected hip fracture risk, especially when the follow-up length was less than 13 years. Although our meta-analysis has provided insufficient evidence that coffee consumption significantly increases hip fracture risk, coffee intake may increase hip fracture risk among women, elderly participants and Northern Americans. No dose-response pattern was observed.

Key words: Coffee consumption: Coffee intake: Hip fracture: Prospective cohort studies: Case-control studies: Meta-analyses

Accompanied by a rapid increase in incidence in recent decades, hip fracture, the most serious complication of osteoporosis, has become a major health problem in many countries ${ }^{(1)}$, particularly among women. Individuals with hip fracture will become a huge burden to society and individuals ${ }^{(2,3)}$, and will be expected to experience poor life quality. Furthermore, it is estimated that there could be 4.5 million hip fractures by $2050^{(4)}$, so it is imperative to identify risk factors of hip fracture followed by developing preventive strategies.

Previous research has demonstrated that decreased bone mineral density in the proximal femur, low $\mathrm{BMI}^{(5)}$, low mobility $^{(6)}$, impaired balance ${ }^{(7)}$ and low physical activity ${ }^{(8)}$ could potentially influence fracture risk. Meanwhile, lifestyle-related extra skeletal risk factors, such as dietary factors, also have a tight relationship with hip fracture. As a common beverage, coffee consumption is high at present, and previous studies have suggested that coffee could increase excretion of urinary and faecal $\mathrm{Ca}^{(5)}$, decrease the efficiency of intestinal Ca absorption $^{(9)}$ and result in Ca loss of about 4-6 mg per cup of coffee. It has been hypothesised that coffee consumption could increase individual susceptibility to fracture by decreasing bone mineral density and bone mechanical strength, but results from earlier research have been conflicting. A total of eight case-control studies ${ }^{(5,10-16)}$ and twelve prospective cohort studies $^{(6,17-27)}$ have explored associations between coffee

* Corresponding author: Dr Xin-li Li, fax +86 5126588 3323, email lixinli@suda.edu.cn

† Both authors contributed equally.

(C) The Author(s) 2013. The online version of this article is published within an Open Access environment subject to the conditions of the Creative Commons Attribution-NonCommercial-ShareAlike licence <http://creativecommons.org/licenses/by-nc-sa/2.5/>. The written permission of 
consumption and hip fracture risk, among which eight studies showed positive association ${ }^{(6,11,18,21-24,27)}$ and one study in Sweden found that coffee consumption could increase hip fracture risk for non-drinkers ${ }^{(19)}$. Another study in Norway showed that women with an intake of at least nine cups of coffee daily tended to have an increased risk of hip fracture, but only $6.8 \%$ of women consumed so much coffee per $\mathrm{d}^{(20)}$. However, results from three studies, conducted in the Netherlands ${ }^{(17)}$, Finland ${ }^{(25)}$ and southern California ${ }^{(26)}$, respectively, showed that coffee consumption had no association with hip fracture.

In view of the inconsistency of the published literatures, a meta-analysis was conducted to investigate the following: (1) to establish the current evidence concerning the relationship between coffee consumption and hip fracture risk; (2) to analyse the relationship between coffee consumption and the risk of hip fracture according to study design and characteristics of study populations; and (3) to determine the potential dose-response pattern between coffee consumption and hip fracture risk.

\section{Methods}

\section{Literature search}

The PubMed database (http://www.ncbi.nlm.nih.gov/entrez/ query.fcgi) was screened through to November 2011 by using the following key words of 'coffee consumption' or 'coffee intake' and 'hip fracture', with restriction for English-language articles only. Reference lists of retrieved articles, reviews and the PubMed option 'related articles' were also searched for potentially relevant papers. Only published studies with full text were included, while abstracts or unpublished studies were excluded. We did not contact authors to obtain additional data.

\section{Inclusion criteria}

Studies matching the following criteria were included in our final meta-analysis: (1) prospective cohort studies or casecontrol studies; (2) research factors were coffee consumption and hip fracture; (3) endpoint for prospective cohort studies was the incidence of hip fracture; (4) association of coffee consumption and hip fracture risk was evaluated, OR or relative risk, and the corresponding $95 \%$ CI for the highest compared with the lowest category of coffee consumption were available.

\section{Data extraction}

Data were collected in a standardised data-collection form according to reference ${ }^{(28)}$, such as: (1) key components of study designs, including first author's surname, publication year, study location, number and age of cases and controls for case-control studies, or number and age of cases and total participants, mean duration of follow-up for prospective cohort studies; (2) evaluation of exposure and outcome, such as dose of coffee consumption, OR or relative risk from the most fully adjusted model for the highest $v$. the lowest coffee consumption and their corresponding $95 \% \mathrm{CI}$; and (3) confounders adjusted for in multivariate analysis. Coffee consumption was expressed uniformly as cups/d.

\section{Quality assessment of studies}

As suggested by Stroup et al. ${ }^{(28)}$, key components of study designs, but not the aggregate scores, were employed to assess the quality of individual studies in our meta-analysis, which included characteristics of study populations, exposure and outcome, duration of follow-up for prospective studies, and potential confounding factors. Literature search, study selection and data extraction were conducted by X.-L. L. and J.-H. X. independently. Discrepancies were discussed.

\section{Statistical methods}

OR was used to assess the association between coffee consumption and hip fracture risk, and relative risk was directly considered as OR when the incidence of hip fracture was low.

The heterogeneity assumption across studies was tested by a $\chi^{2}$-based Q test. A P value of greater than $0 \cdot 10$ indicated a lack of heterogeneity. The $I^{2}$ statistic, which describes the proportion of total variation in study estimates caused by heterogeneity $^{(29)}$, was also calculated. If the heterogeneity test was statistically significant, the random-effects model (DerSimonian and Laird method) was employed. Otherwise, the pooled estimation of the OR was calculated by the fixed-effects model (Mantel-Haenszel method) ${ }^{(30)}$.

Effects of various study characteristics, including study design, case sex, case age, geographic regions, publication year of the paper, and length of follow-up for prospective cohort study were investigated in subgroup analyses. The cutpoint of follow-up duration was determined according to the median length of follow-up. As most of the datasets (eleven of fourteen) used in the present analysis were published before the year 2000, and the amount of caffeine in commercial coffee products, lifestyle, and other risk factors of hip fracture may be different from the past decades, the data were reanalysed on the basis of publication year 2000.

A sensitivity analysis was performed using both fixed- and random-effects models to evaluate the influence of single study on the overall risk, by omitting one study each time.

Potential publication bias was assessed by Begg's funnel plots and Egger's regression test $(P<0 \cdot 10)^{(31)}$, an asymmetric plot suggests possible publication bias.

All analyses were performed by using STATA version $11 \cdot 0$ (StataCorp LP). A P value $<0.05$ was considered significant, except as where otherwise specified.

\section{Results}

\section{Eligible studies}

Our initial search by using 'coffee consumption' OR 'coffee intake' AND 'hip fracture' obtained twenty-one eligible studies in PubMed, and six studies were added after reviewing the reference lists of retrieved articles, reviews and the PubMed option 'related articles'. The full text of the total articles 
were reviewed, and fifteen studies were excluded for the following reasons: two studies were reviews ${ }^{(32,33)}$, eight studies could not provide the $\mathrm{OR}$ and their $\mathrm{CI}^{(6,15,16,23-27)}$, and five studies had irrelevant endpoints and exposures ${ }^{(34-38)}$. The remaining twelve studies included six case-control studies $^{(5,10-14)}$ and six prospective studies ${ }^{(1-22)}$, among which two studies ${ }^{(20,22)}$ reported their results separately by sex, and thus each study was regarded as two separate studies, so fourteen datasets were extracted. The selection progress of papers is displayed in Fig. 1.

\section{Study characteristics}

The characteristics of the included studies are displayed in Tables 1 and 2. All the studies were published between 1990 and 2011, and seven studies were conducted in Europe, four in North America and one in Asia. They were all population-based except the Nurses' Health Study ${ }^{(21)}$. The median length of follow-up for prospective studies was $13 \cdot 3$ years. Coffee consumption was assessed by using a semiquantitative dietary questionnaire. Case ascertainments were confirmed by using hospital discharge registers or individual medical records or telephone for eleven studies; only one was self-report fractures ${ }^{(21)}$. All the OR in each study were estimated based on the highest compared with the lowest coffee consumption. Most individual studies were adjusted for a wide range of potential confounders, including age, BMI, physical activity, smoking, alcohol use, energy intake, etc. except for the study of Johnell et al. ${ }^{(5)}$, which was only adjusted for age, research centre and BMI.

\section{Meta-analysis results}

The multivariable-adjusted OR for each study and the pooled OR for the highest compared with the lowest level of coffee consumption are presented in Fig. 2. Of the fourteen groups of datasets, eleven showed positive association and three were negatively associated. Of the eleven studies showing positive association, four studies reached statistical significance $^{(11,18,21,22)}$. Of the three studies showing negative association, only one reached statistical significance ${ }^{(19)}$. Obvious heterogeneity was found across studies $\left(P=0 \cdot 000 ; I^{2}=84 \cdot 0\right.$ $\%)$, and therefore the random-effects model was employed; the pooled OR of hip fracture risk for the highest compared with the lowest coffee consumption was 1.297 (95\% CI $0 \cdot 960,1 \cdot 751 ; P=0 \cdot 09)$.

\section{Subgroup and sensitivity analyses}

The results of subgroup analyses according to study design, case sex, case age, geographic region, and length of follow-up for prospective studies and publication year of the paper are presented in Table 3. Hip fracture risk increased significantly if the coffee drinkers were female, elderly subjects or North American, while study design had no effect on the pooled OR. Subgroup analysis according to data published before the year 2000 demonstrated that coffee drinking significantly increased hip fracture risk by 41.9\% (OR 1.419; $95 \%$ CI $1.074,1.874)$, while results from data published after the year 2000 showed a decreased risk of hip fracture by $9.7 \%$ (OR 0.903; $95 \%$ CI 0.473, 1.866). Results regarding the effect of follow-up length on hip fracture risk showed that when the

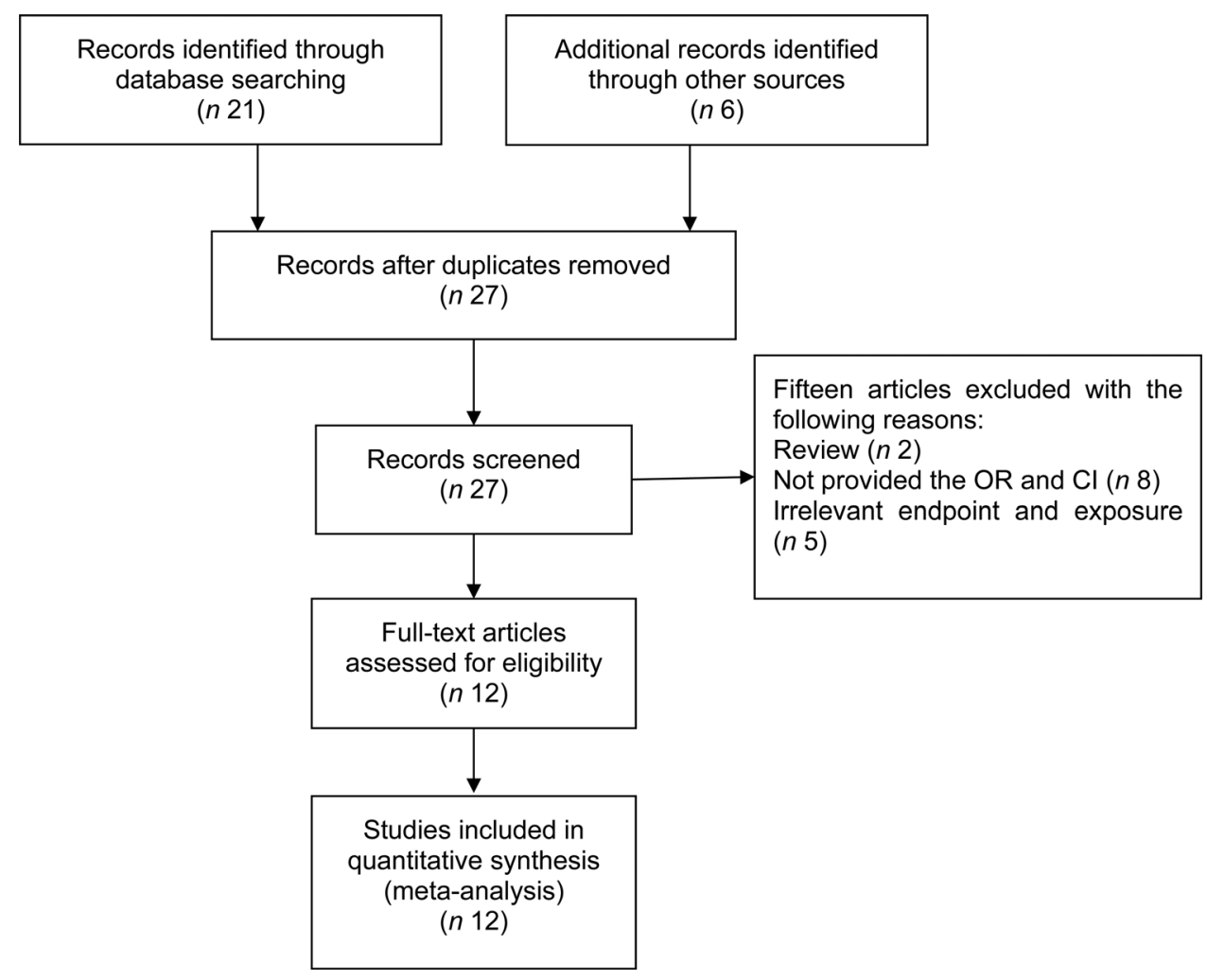

Fig. 1. Flow diagram of study selection. 


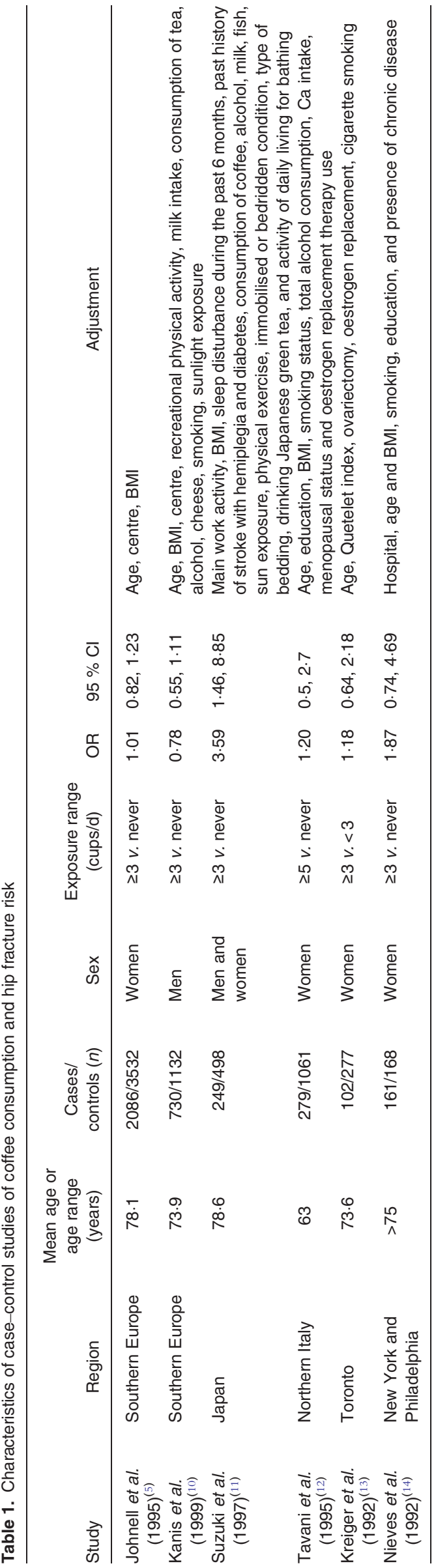

length of follow-up was more than 13 years, hip fracture risk significantly decreased by $41.8 \%$ (OR 0.582; $95 \%$ CI 0.453, $0 \cdot 747$ ), while when the follow-up was no more than 13 years, the risk significantly increased by $71.2 \%$ (OR 1.712; $95 \%$ CI $1 \cdot 517,1.931)$.

The sensitivity analysis conducted by omitting one study each time and calculating the combined OR for the remaining studies was perfornmed. All other trials had no substantial effect on hip fracture risk (from 1.153 (95\% CI 0.853, $1.559)$ to $1.343(95 \%$ CI $0.944,1.911)$ ) except the study by Trimpou et al. ${ }^{(19)}$, which resulted in significantly increased hip fracture risk by 38.9 \% (OR 1.389; $95 \%$ CI 1.068, 1.806).

\section{Dose-response analysis}

Although several included studies ${ }^{(5,10-12,14,17,19-22)}$ had stratified their results by dose of coffee consumption, only four studies $^{(19-22)}$ (five datasets) could be selected to conduct final dose-response analysis, while others were excluded for not providing participant number of each category of coffee consumption. The dose-response analysis showed a slight, but not significant, reduction in hip fracture risk by $0.3 \%$ for every cup/d increment in coffee intake (OR 0.997; $95 \%$ CI 0.964, 1.031; $P$ for trend $=0.872$ ).

\section{Publication bias}

Publication bias was tested by Begg's funnel plot and Egger's test. Result of Begg's funnel plot did not show significant asymmetry (Fig. 3), and Egger's test also had little evidence of publication bias $(P=0 \cdot 891)$.

\section{Discussion}

Our present meta-analysis firstly evaluates the effect of coffee intake on hip fracture risk. Meta-analysis results showed a slightly, although not significantly, increased risk of hip fracture by $29.7 \%$ (OR 1.297; $95 \%$ CI 0.960, 1.751; $P=0.09$ ) in response to coffee intake. Obvious heterogeneity was displayed. The dose-response relationship, only four studies included $^{(19-22)}$, showed a slight reduction of hip fracture risk by $0.3 \%$ (OR 0.997; $95 \%$ CI 0.964, 1.031; $P$ for trend $=$ $0 \cdot 872$ ) in response to every cup/d increment of coffee consumption, but the results had no statistical significance.

The heterogeneity might be explained by the following. First, studies included in our meta-analysis were conducted in different geographic regions, where populations shared different genetic background, lifestyle and hip fracture incidence. Second, the sex and age of the participants varied narrowly.

Results from subgroup analyses demonstrated that female coffee drinker, elderly participants and North Americans were at higher risk of hip fracture, which is consistent with previous studies ${ }^{(18,21,22,39)}$. Elderly women have different hormone levels, which could affect the metabolism of Ca. The region of America had the highest incidence rate of hip fracture $^{(39)}$, which could be explained by the higher caffeine content of coffee than that of other regions, such as Italy ${ }^{(12)}$. 


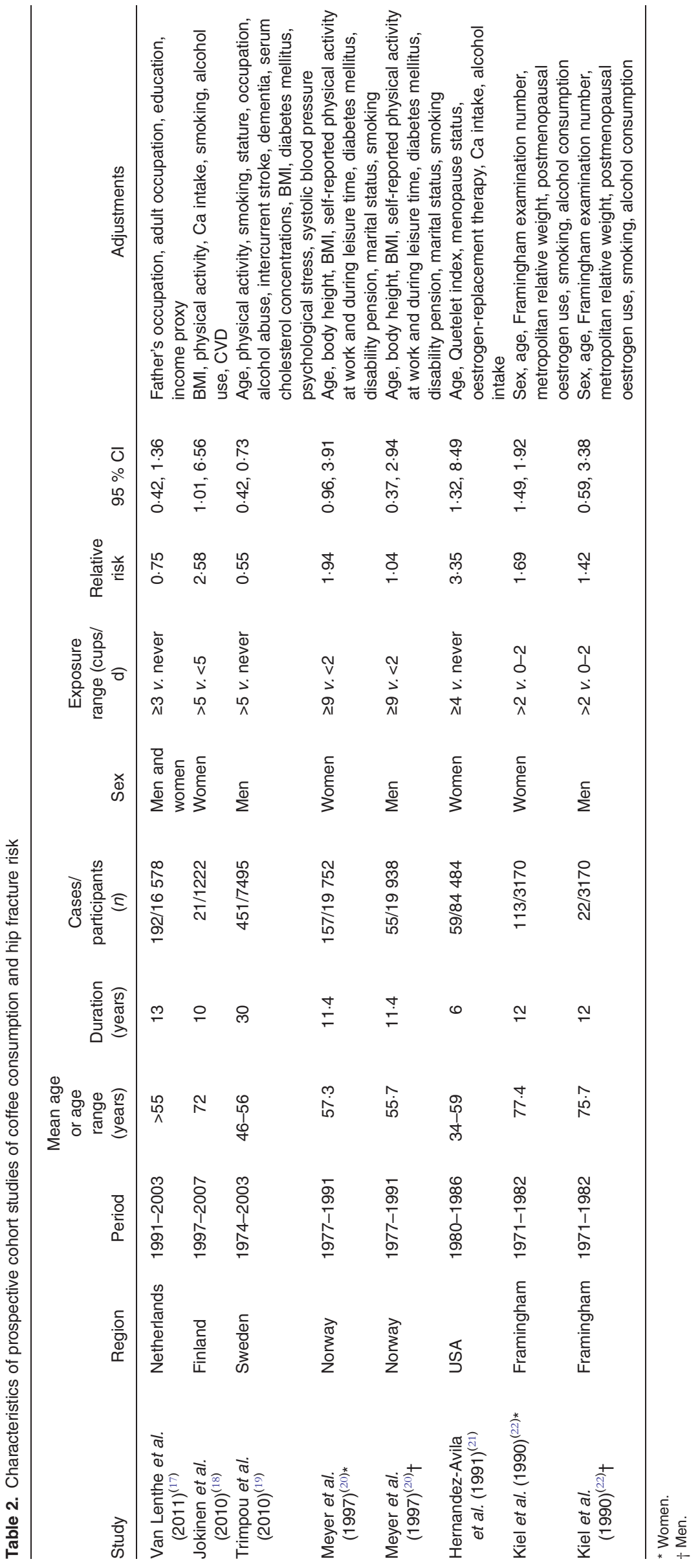


Study

OR or RR $(95 \% \mathrm{Cl})$

Weight

Johnell et al. (1995) ${ }^{(5) *}$

Kanis et al. (1999) $)^{(10) *}$

Suzukiet al. (1997)(11)*

Tavani et al. (1995) ${ }^{(12) *}$

Kreiger et al. (1992)(13)*

Nieves et al. (1992) ${ }^{(14) *}$

Van Lenthe et al. (2011)(17)†

Jokinen et al. $(2010)^{(18)} \dagger$

Trimpou et al. (2010) ${ }^{(19)} \dagger$

Meyer et al. (1997) ${ }^{(20)} \dagger$ women

Meyer et al. $(1997)^{(20)} \dagger$ men

Hernandez-Avila et al. (1991)(21) $†$

Kiel et al. (1990)(22) † women

Kiel et al.1990(22) $†$ men

Overall ( $\left.I^{2} 84.5 \% ; P=0.000\right)$

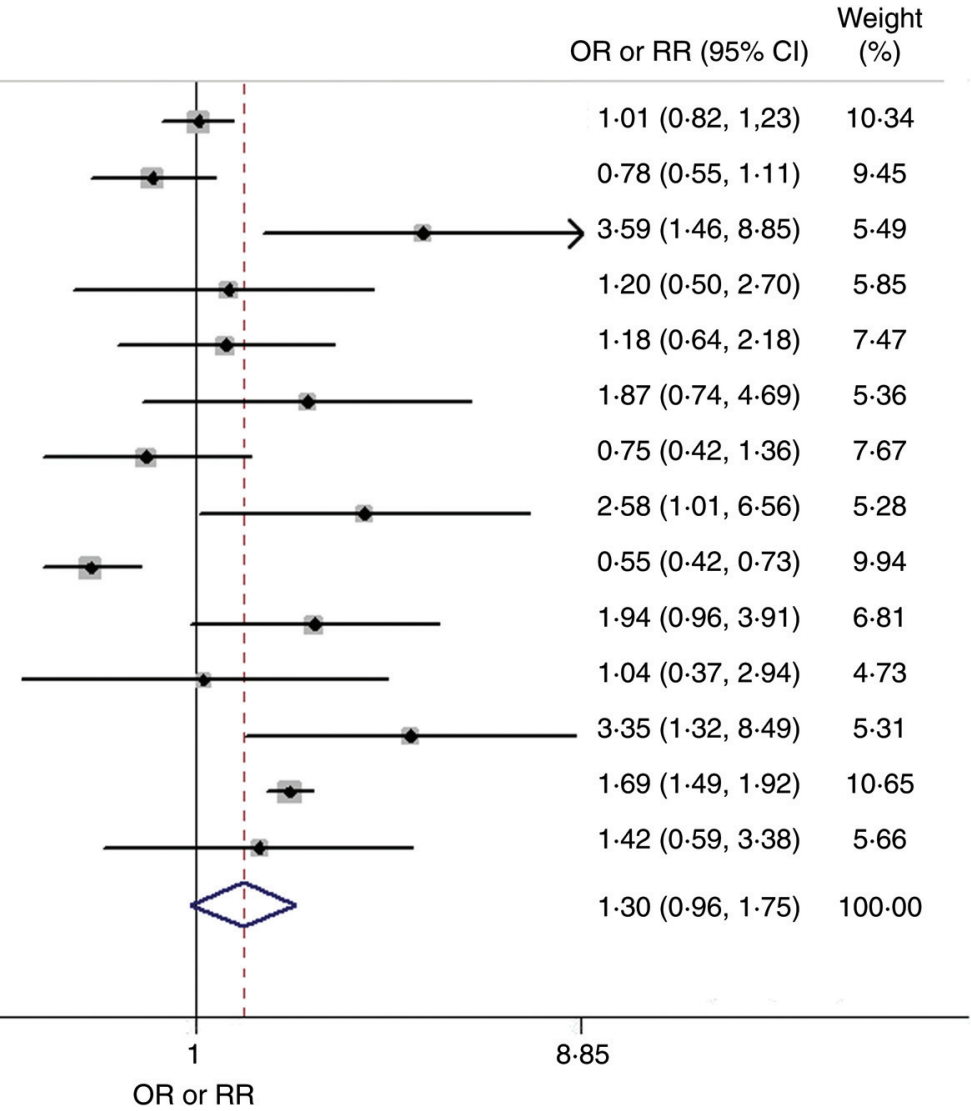

Fig. 2. Meta-analysis of studies that investigated association of coffee consumption with hip fracture risk (random-effects model). ${ }^{*}$ OR. $\dagger$ Relative risk (RR).

Further subgroup analysis showed that follow-up duration was negatively associated with hip fracture risk. Among those studies where the duration of follow-up was less than 13 years, coffee consumption was commonly more than five cups/d, while other studies where the length of follow-up was more than 13 years, the median coffee consumption was less than two cups/d. Therefore, it might be the relatively higher coffee consumption not the shorter length of follow-up that induced the above results. Subgroup analysis stratified by the publication year of papers demonstrated that coffee intake increased hip fracture risk according to the data published before the year 2000, while decreased hip fracture risk using

Table 3. Association of coffee intake and hip fracture stratified by certain variables

\begin{tabular}{|c|c|c|c|c|c|}
\hline Variable & Total studies included $(n)$ & Summary relative risk & $95 \% \mathrm{Cl}$ & $P$ for heterogeneity & $I^{2}(\%)$ \\
\hline All & 14 & 1.297 & $0.960,1.751$ & 0 & 84.0 \\
\hline \multicolumn{6}{|l|}{ Study design } \\
\hline Prospective cohort studies & 8 & 1.357 & $0.822,2.240$ & 0 & 88.9 \\
\hline Case-control studies & 6 & $1 \cdot 181$ & $0.889,1.569$ & 0.078 & 49.5 \\
\hline \multicolumn{6}{|l|}{ Sex } \\
\hline Women & 8 & 1.547 & $1 \cdot 152,2.077$ & 0.001 & 70.5 \\
\hline Men & 4 & 0.752 & $0.523,1.080$ & 0.102 & 51.7 \\
\hline Men and women & 2 & 1.578 & $0.341,7.309$ & 0.004 & $87 \cdot 7$ \\
\hline \multicolumn{6}{|l|}{ Age } \\
\hline$>70$ years & 8 & 1.401 & $1.015,1.935$ & 0 & $80 \cdot 3$ \\
\hline $50-70$ years & 6 & 1.145 & $0.645,2.032$ & 0 & 78.6 \\
\hline \multicolumn{6}{|l|}{ Geographic region } \\
\hline Europe & 8 & 0.970 & $0.710,1.325$ & 0.001 & 70.5 \\
\hline North America & 5 & 1.683 & $1.492,1.899$ & 0.464 & 0 \\
\hline Asia & 1 & 3.590 & $1.458,8.839$ & & \\
\hline \multicolumn{6}{|l|}{ Length of follow-up } \\
\hline$>13$ years & 2 & 0.582 & $0.453,0.747$ & 0.349 & 0 \\
\hline$\leq 13$ years & 6 & 1.712 & $1.517,1.931$ & 0.555 & 0 \\
\hline \multicolumn{6}{|l|}{ Publication year of the paper } \\
\hline Before 2000 & 11 & 1.419 & $1.074,1.874$ & 0.001 & 79.9 \\
\hline After 2000 & 3 & 0.903 & $0.473,1.866$ & 0 & $74 \cdot 1$ \\
\hline
\end{tabular}




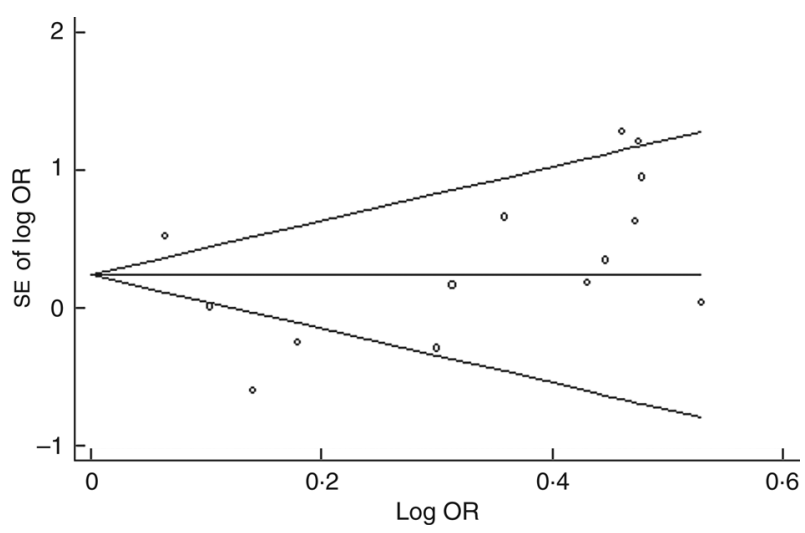

Fig. 3. Funnel plot of coffee consumption and risk of hip fracture.

data published after year 2000; the change in caffeine content caused by a change in method of coffee preparation or cup size might account for the above results ${ }^{(40)}$.

In the sensitivity analysis, the study by Trimpou et al. ${ }^{(19)}$ had a significant effect on the pooled OR. Trimpou's study ${ }^{(19)}$ is the only one included in our final meta-analysis that showed a significant negative association between coffee intake and hip fracture, with the combined risk of 0.55 (95\% CI 0.42, $0.73)$. Its participants were all male, and were classified into two categories: non-drinkers and coffee drinkers. The author further testified that those non-drinkers, with a significantly increased risk $(P=0.0002)$ of hip fractures, often suffered from digestive tract problems; thus, it might be the digestive tract problems that increased hip fracture risk, not the coffee that protected the drinkers from hip fracture risk. Meanwhile, the participants in Trimpou's study ${ }^{(19)}$ were relatively young, which might be one reason for the increased pooled OR.

In addition to the variables included in the present study, there are other factors that should be considered. Method of coffee preparation, type of coffee, such as black coffee, coffee with milk, or coffee with cream determine the caffeine content. The menopausal status of women and differences in genetic background, such as genetic polymorphism in the NAT2 gene $^{(41)}$, could affect the metabolism of caffeine and caffeine exposure of an individual. Different subtypes of hip fractures had different responses to coffee, and only trochanteric fracture was significantly associated with heavy coffee consumption $^{(42)}$. Dietary factors, such as intake of Ca and vitamin D, could affect $\mathrm{Ca}$ balance. Protein from non-dairy animal sources is likely to affect $\mathrm{Ca}$ excretion ${ }^{(43)}$. Although several studies $^{(12,13,20)}$ had demonstrated that low Ca intake and short sunlight exposure ${ }^{(5,10,11)}$ could increase hip fracture risk, only limited studies had stratified their results according to the level of $\mathrm{Ca}$ intake ${ }^{(12)}$ or menopausal status ${ }^{(12,13)}$. At present, as the selected papers in the present review could not provide more available evidence about the above factors, we failed to further analyse the effect of those factors on the relationship between coffee and hip fracture, and research concerning those factors is needed in the future.

The present review has several limitations that should be considered. First, obvious heterogeneity was observed.
Therefore, the random-effects model was employed. Second, exposure misclassification should be considered. Coffee exposure is expressed by cups/d, and assessed using FFQ. This might introduce some degree of error in the exposure measurement. For example, the cup size and the caffeine content of the same commercial coffee products may differ materially between different studies. Nevertheless, the total cups of coffee intake per $\mathrm{d}$ at least gave us a hint about the relationship between coffee intake and hip fracture risk. Third, although most included studies adjusted for a number of potential confounders for hip fracture, it may be possible that other unmeasured or inadequately measured factors have confounded the true association of coffee intake and hip fracture. Fourth, all the studies except one ${ }^{(11)}$ in our analysis were conducted in Western countries; additional research in other populations is warranted to generalise the findings.

However, there are several advantages of the present study. First, we had included twelve studies that were mainly population-based except for the study of Tavani et al. ${ }^{(12)}$, which was hospital-based; this could greatly reduce the likelihood of selection biases. Second, most of the trials (eleven of twelve) were conducted in Europe and America, where individuals shared a similar genetic sensitivity to caffeine, and the effect of genetic variation on the caffeine exposure of an individual was small. Third, the present study had collected a large number of cases, so the present results had a highly sufficient statistical power. The mean follow-up of 13.3 years of the prospective cohort studies was sufficiently long to detect an adequate number of hip fractures.

In Western countries, coffee consumption per capita is high, especially in Nordic countries. Meanwhile, hip fracture is considered a major health problem in many countries. Due to the fact that a substantial proportion of the population are daily consumers of coffee (and thus caffeine), and the effect of coffee intake on fracture risk has been conflicting, it is imperative to classify the exact effect of coffee consumption on hip fracture risk. Although our present meta-analysis showed that the role of coffee consumption in increasing hip fracture risk had no statistical significance, coffee intake may increase hip fracture risk for female, elderly coffee drinkers and Northern Americans. Further studies that consider interactions between coffee consumption and dietary factors, lifestyle factors and genetic polymorphisms are warranted to confirm our findings in the future.

\section{Acknowledgements}

The present review was supported by grants from the National Natural Science Foundation of China (no. 81001185) and the Universities Natural Science Foundation of Jiangsu Province (no. 10KJB310011).

X.-L. L. and J.-H. X. were both involved in literature search, study selection and data extraction. J.-H. X. performed the data analyses; and X.-L. L. was involved in writing the manuscript. Both authors participated in the revision of the manuscript.

We declare no conflict of interest. 


\section{References}

1. Chrischilles E, Shireman T \& Wallace R (1994) Costs and health effects of osteoporotic fractures. Bone 15, 377-386.

2. Nurmi I, Narinen A, Luthje P, et al. (2003) Cost analysis of hip fracture treatment among the elderly for the public health services: a 1 -year prospective study in 106 consecutive patients. Arch Orthop Trauma Surg 123, 551-554.

3. Kanis JA, Odén A, Johnell O, et al. (2003) The components of excess mortality after hip fracture. Bone 32, 468-473.

4. Gullberg B, Johnell O \& Kanis JA (1997) World-wide projections for hip fracture. Osteoporos Int 7, 407-413.

5. Johnell O, Gullberg B, Kanis JA, et al. (1995) Risk factors for hip fracture in European women: the MEDOS Study. Mediterranean Osteoporosis Study. J Bone Miner Res 10, 1802-1815.

6. Cummings SR, Nevitt MC, Browner WS, et al. (1995) Risk factors for hip fracture in white women. Study of Osteoporotic Fractures Research Group. N Engl J Med 332, 767-773.

7. Wagner H, Melhus H, Gedeborg R, et al. (2009) Simply ask them about their balance - future fracture risk in a nationwide cohort study of twins. Am J Epidemiol 169, 143-149.

8. Gregg EW, Cauley JA, Seeley DG, et al. (1998) Physical activity and osteoporotic fracture risk in older women. Study of Osteoporotic Fractures Research Group. Ann Intern Med 129, 81-88.

9. Barger-Lux MJ \& Heaney RP (1995) Caffeine and the calcium economy revisited. Osteoporos Int 5, 97-102.

10. Kanis J, Johnell O, Gullberg B, et al. (1999) Risk factors for hip fracture in men from southern Europe: the MEDOS study. Mediterranean Osteoporosis Study. Osteoporos Int 9, 45-54.

11. Suzuki T, Yoshida H, Hashimoto T, et al. (1997) Case-control study of risk factors for hip fractures in the Japanese elderly by a Mediterranean Osteoporosis Study (MEDOS) questionnaire. Bone 21, 461-467.

12. Tavani A, Negri E \& La Vecchia C (1995) Coffee intake and risk of hip fracture in women in northern Italy. Prev Med 24, 396-400.

13. Kreiger N, Gross A \& Hunter G (1992) Dietary factors and fracture in postmenopausal women: a case-control study. Int J Epidemiol 21, 953-958.

14. Nieves JW, Grisso JA \& Kelsey JL (1992) A case-control study of hip fracture: evaluation of selected dietary variables and teenage physical activity. Osteoporos Int 2, 122-127.

15. Cumming RG \& Klineberg RJ (1994) Case-control study of risk factors for hip fractures in the elderly. Am J Epidemiol 139, 493-503.

16. Michaëlsson K, Holmberg L, Mallmin H, et al. (1995) Diet and hip fracture risk: a case-control study. Study Group of the Multiple Risk Survey on Swedish Women for Eating Assessment. Int $J$ Epidemiol 24, 771-782.

17. Van Lenthe FJ, Avendano M, van Beeck EF, et al. (2011) Childhood and adulthood socioeconomic position and the hospitalbased incidence of hip fractures after 13 years of follow-up: the role of health behaviours. J Epidemiol Community Health 65, 980-985.

18. Jokinen H, Pulkkinen P, Korpelainen J, et al. (2010) Risk factors for cervical and trochanteric hip fractures in elderly women: a population-based 10-year follow-up study. Calcif Tissue Int 87, 44-51.

19. Trimpou P, Landin-Wilhelmsen K, Odén A, et al. (2010) Male risk factors for hip fracture - a 30-year follow-up study in 7,495 men. Osteoporos Int 21, 409-416.

20. Meyer HE, Pedersen JI, Løken EB, et al. (1997) Dietary factors and the incidence of hip fracture in middle-aged Norwegians. A prospective study. Am J Epidemiol 145, 117-123.

21. Hernandez-Avila M, Colditz GA, Stampfer MJ, et al. (1991) Caffeine, moderate alcohol intake, and risk of fractures of the hip and forearm in middle-aged women. Am J Clin Nutr 54, 157-163.

22. Kiel DP, Felson DT, Hannan MT, et al. (1990) Caffeine and the risk of hip fracture: the Framingham Study. Am J Epidemiol 132, 675684.

23. Trimpou P, Odén A, Simonsson T, et al. (2011) High serum total cholesterol is a long-term cause of osteoporotic fracture. Osteoporos Int 22, 1615-1620.

24. Hallström H, Wolk A, Glynn A, et al. (2006) Coffee, tea and caffeine consumption in relation to osteoporotic fracture risk in a cohort of Swedish women. Osteoporos Int 17, 1055-1064.

25. Huopio J, Kröger H, Honkanen R, et al. (2000) Risk factors for perimenopausal fractures: a prospective study. Osteoporos Int 11, 219-227.

26. Holbrook TL, Barrett-Connor E \& Wingard DL (1988) Dietary calcium and risk of hip fracture: 14-year prospective population study. Lancet ii, 1046-1049.

27. Hansen SA, Folsom AR, Kushi LH, et al. (2000) Association of fractures with caffeine and alcohol in postmenopausal women: the Iowa Women's Health Study. Public Health Nutr 3, 253-261.

28. Stroup DF, Berlin JA, Morton SC, et al. (2000) Meta-analysis of observational studies in epidemiology: a proposal for reporting. Meta-analysis Of Observational Studies in Epidemiology (MOOSE) group. JAMA 283, 2008-2012.

29. Higgins JP, Thompson SG, Deeks JJ, et al. (2003) Measuring inconsistency in meta-analyses. BMJ 327, 557-560.

30. DerSimonian R \& Laird N (1986) Meta-analysis in clinical trials. Control Clin Trials 7, 177-188.

31. Egger M, Davey Smith G, Schneider M, et al. (1997) Bias in meta-analysis detected by a simple, graphical test. BMJ 315, 629-634.

32. Higdon JV \& Frei B (2006) Coffee and health: a review of recent human research. Crit Rev Food Sci Nutr 46, 101-123.

33. Diddle AW \& Smith IQ (1984) Postmenopausal osteoporosis: the role of estrogens. South Med J 77, 868-874.

34. Tanaka S, Narusawa K, Onishi H, et al. (2011) Lower osteocalcin and osteopontin contents of the femoral head in hip fracture patients than osteoarthritis patients. Osteoporos Int 22, 587-597.

35. Fujiwara S, Kasagi F, Yamada M, et al. (1997) Risk factors for hip fracture in a Japanese cohort. J Bone Miner Res 12, 998-1004.

36. Brown SL, Salive ME, Pahor M, et al. (1995) Occult caffeine as a source of sleep problems in an older population. J Am Geriatr Soc 43, 860-864.

37. Carson CA, Cauley JA \& Caggiula AW (1993) Relation of caffeine intake to blood lipids in elderly women. Am J Epidemiol 138, 94-100.

38. Kiel DP, Baron JA, Anderson JJ, et al. (1992) Smoking eliminates the protective effect of oral estrogens on the risk for hip fracture among women. Ann Intern Med 116, 716-721.

39. Villa ML \& Nelson L (1996) Race, ethnicity, and osteoporosis. In Osteoporosis, pp. 435-437 [R Marcus, D Felman and JL Kelsey, editors]. San Diego: Academic.

40. McCusker RR, Goldberger BA \& Cone EJ (2003) Caffeine content of specialty coffees. I Anal Toxicol 27, 520-522.

41. Carrillo JA \& Benitez J (2000) Clinically significant pharmacokinetic interactions between dietary caffeine and medications. Clin Pharmacokinet 39, 127-153.

42. Grisso JA, Kelsey JL, Strom BL, et al. (1994) Risk factors for hip fracture in black women. New Engl J Med 330, 1555-1559.

43. Hu JF, Zhao XH, Parpia B, et al. (1993) Dietary intakes and urinary excretion of calcium and acids: a cross-sectional study of women in China. Am J Clin Nutr 58, 398-406. 